\title{
PERCEPCIÓN DE CALIDAD DE LOS PROGRAMAS DE ENTRETENIMIENTO TELEVISIVOS EN ESPAÑA: INFLUENCIA DE LOS VALORES DE PRODUCCIÓN
}

\author{
Quality perception of entertainment TV shows in \\ Spain: production values
}

Enrique Guerrero y Cristina Etayo

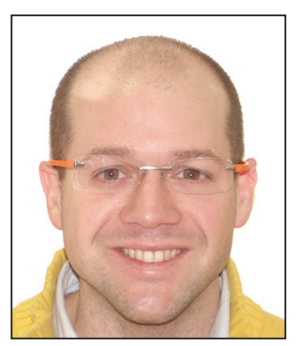

Enrique Guerrero es doctor en comunicación audiovisual (2009) por la Universidad de Navarra y cursó el Certificate in entertainment and media management (2007) de la University of California, Los Ángeles (UCLA). Su investigación se centra en el estudio de los estándares de producción de los contenidos de entretenimiento en televisión y en el impacto de la digitalización en la industria audiovisual. Ha sido investigador visitante de la Bournemouth University (2011) y de la University of Texas en Austin (2013).

http://orcid.org/0000-0001-7693-8669

Universidad de Navarra, Departamento de Cultura y Comunicación Audiovisual Campus Universitario. Edif. Biblioteca de Humanidades. 31009 Pamplona, España eguerrero@unav.es

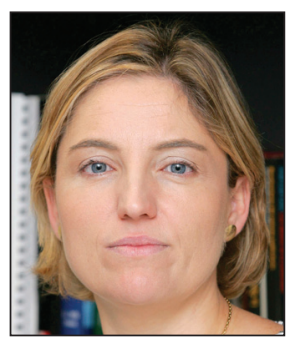

Cristina Etayo es profesora titular de la Facultad de Comunicación de la Universidad de Navarra (España). Ha desarrollado su investigación en el área de los medios de comunicación, especialmente sobre publicidad en televisión. Trabaja en un proyecto sobre los nuevos hábitos de consumo audiovisual en Europa, donde analiza el impacto de la digitalización en el consumo y en la industria.

http://orcid.org/0000-0002-8464-0691

Universidad de Navarra, Departamento Empresa Informativa Campus Universitario. Edif. Biblioteca de Humanidades. 31009 Pamplona, España cetayo@unav.es

\section{Resumen}

Se analiza qué valores de producción tienen mayor impacto en la percepción de calidad que la audiencia tiene respecto a los diferentes géneros de entretenimiento televisivos. Para ello se han seleccionado los siguientes valores de producción: contenido, presentador/a, decorado y calidad creativa y técnica. La principal fuente de información ha sido una encuesta telefónica propia dirigida a una muestra representativa de la población española. Los resultados confirman que, a pesar de los cambios radicales vividos por el sector y el boom de la televisión conectada (como resultado de la convergencia entre televisión e internet), la clave del negocio es la misma de siempre: la calidad del contenido.

\section{Palabras clave}

Televisión; Entretenimiento; Valores de producción; Calidad; Audiencias; Encuestas.

\begin{abstract}
The aim of this paper is to analyse which production values are more determinant regarding the audience's quality perception with respect to different types of entertainment television genres. In order to develop this study, we have taken into account production values such as the creative and technical quality, the host, the set and the content. The main source of data for this empirical study is an original survey. The target population consisted of the Spanish population and the sample was designed to be representative. The results show that in spite of the emergence of the connected TV -resulting from the convergence of television and the internet-, which is profoundly changing the audiovisual market, the cornerstone of their business remains the same: content quality.
\end{abstract}

\section{Keywords}

Television; Entertainment; Production values; Quality; Audiences; Surveys. 
Guerrero, Enrique; Etayo, Cristina (2015). "Percepción de calidad de los programas de entretenimiento televisivos en España: influencia de los valores de producción”. El profesional de la información, v. 24, n. 3, pp. 256-264.

http://dx.doi.org/10.3145/epi.2015.may.05

\section{Introducción}

La televisión está sumida en una intensa época de cambios debido a la digitalización, la convergencia con internet y el acceso a contenidos audiovisuales mediante pantallas móviles en cualquier lugar y momento. Los cambios derivados de la innovación tecnológica han revolucionado el sector desde múltiples perspectivas: producción de contenidos, distribución y comercialización, modelos de negocio, hábitos de visionado de la audiencia, etc. No obstante, a pesar de las profundas transformaciones vividas por el medio desde su nacimiento, permanece abierto un debate que parece inmune a las mejoras tecnológicas: la eterna discusión en torno a la calidad de sus contenidos.

Algunas investigaciones previas advierten que el crecimiento de oferta y consumo televisivo no se corresponden con una mejora proporcional del índice de satisfacción y la percepción de calidad por parte de la audiencia (Artero; Herrero; Sánchez-Tabernero, 2010). Sin embargo conviene distinguir por una parte entre calidad medida según criterios objetivos y percepción de calidad según criterios subjetivos-, y por otra, entre percepción y satisfacción. En este artículo abordamos qué valores de producción tienen mayor impacto en la percepción de calidad que los telespectadores tienen sobre los programas de entretenimiento. En concreto nos centramos en los siguientes géneros: concursos, magazines y talk shows, telerrealidad, programas musicales y de búsqueda de talentos, programas de entretenimiento y humor, divulgativos y docuseries ${ }^{1}$.

Se parte de la premisa de que la calidad es relativa al género y que por tanto es posible encontrar en la programación contenidos de entretenimiento de calidad (Guerrero, 2005; Mir et al., 2008; Medina, 2009). Más aún, dada la naturaleza de la televisión como medio, es imprescindible que el resto de géneros también entretengan para que puedan cumplir adecuadamente la misión para la que fueron producidos. La cualidad de "entretener", entendida como la capacidad de un contenido para "distraer la atención de la mente en situaciones que aporten algo" (Medina, 2009), destaca no sólo como un criterio de calidad, sino como un requisito para la televisión generalista o dirigida a grandes audiencias. Los programas de entretenimiento, aunque sólo entretengan, cumplen una función social y presentan varios grados de calidad en función de su valor público (Von-Rimscha; De-Acevedo; Siegert, 2010).

Los valores de producción están vinculados al estándar de producción, una serie de parámetros creativos, técnicos y de organización asociados a tres variables que determinan su nivel de calidad: tiempo, coste y calidad (Diego; Pardo, 2008). Los profesionales del medio los identifican con indicios visibles que están presentes en los programas de cali- dad y que permiten diferenciar los contenidos respecto a la competencia (Cummins; Chambers, 2011).

En esta investigación analizamos cuatro valores de producción relevantes:

- contenido;

- presentador/a;

- decorado;

- calidad creativa y técnica.

Se han seleccionado siguiendo el doble criterio de visibilidad y presupuesto: todos son visibles y reconocibles por la audiencia sin necesidad de contar con formación profesional, y representan un elevado porcentaje del presupuesto de las producciones televisivas. En la mayor parte de proyectos de entretenimiento para televisión, en torno al $80 \%$ del presupuesto se destina al personal creativo, artístico y técnico, a los medios técnicos y a la escenografía y dirección artística (Guerrero, 2013). Por tanto es de gran interés para cadenas y productoras determinar si estos valores tienen influencia en la percepción de calidad por parte de la audiencia.

Para valorar la percepción de calidad respecto al contenido e interpretar los resultados en su contexto, se ha identificado una serie de problemas de orden moral y pragmático que la afectan de modo negativo:

a) morales:

- incumplimiento del horario de protección infantil;

- contenidos violentos;

- erotismo y pornografía;

b) pragmáticos:

- exceso de publicidad;

- falta de programación cultural;

- contenidos sobre vida privada de personalidades populares (cotilleo);

- ausencia de variedad en la programación y sensacionalismo.

La hipótesis de partida es que los valores de producción influyen en la percepción de calidad de la audiencia respecto a los programas de entretenimiento, aunque los telespectadores carezcan de conocimiento profesional especializado.

Se parte de estudios precedentes, no en vano la calidad televisiva ha suscitado el interés académico desde los orígenes del medio debido a su influencia social. Su estudio puede abordarse desde diversas perspectivas: tipo de contenido, naturaleza pública o privada de la empresa televisiva, criterio profesional, percepción de la audiencia, regulación, crítica especializada, etc. (Gutiérrez-Gea, 2000; Von-Rimscha; De-Acevedo; Siegert, 2010). Numerosos trabajos de investigación han intentado estandarizar el proceso de medición de la calidad de contenidos audiovisuales mediante pautas 


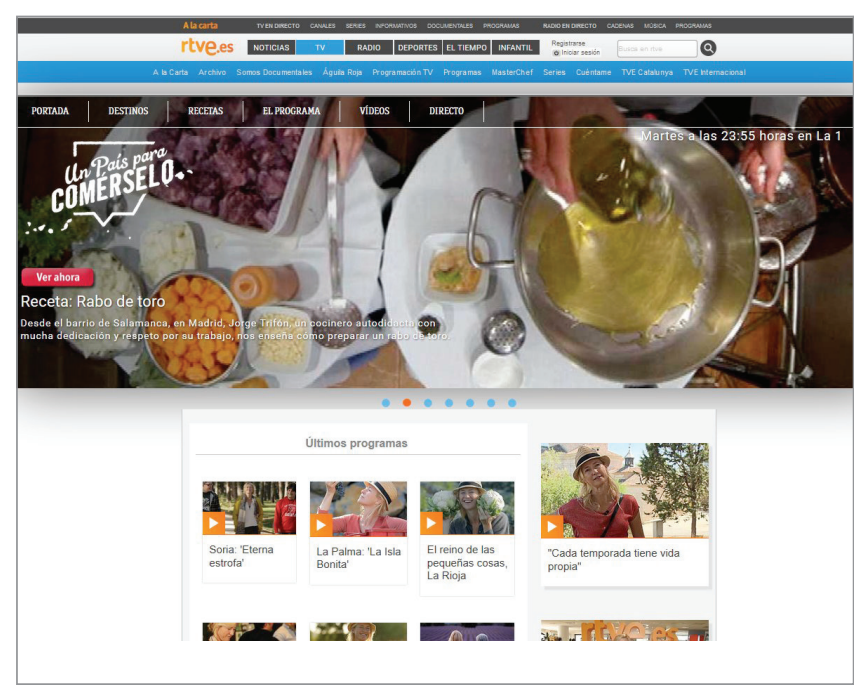

Docuserie. Un país para comérselo, La 1

http://www.rtve.es/television/un-pais-para-comerselo

objetivas, dejando los criterios subjetivos para el análisis de la percepción de la calidad.

Existen dos corrientes. Sánchez-Tabernero (2008) hace explícita esta dualidad al afirmar que la calidad en los medios presenta dos dimensiones:

a) la subjetiva (según la percepción del público);

b) la objetiva (según los estándares profesionales).

Por una parte algunos autores abordan el estudio de la calidad televisiva desde la óptica de los profesionales del medio, por tener el conocimiento técnico y la autoridad para valorar la calidad de los contenidos. Por ejemplo, Lasagni y Richeri (2006) intentan encontrar pautas objetivas de medición de calidad mediante entrevistas a profesionales. Sin embargo, dichos profesionales no parecen compartir un marco de referencia común y universal que permita objetivar unos criterios para indicar que un contenido sea o no de calidad (Albers, 1992). Por otra parte, algunos investigadores consideran necesario incluir también el criterio de la audiencia para identificar las cualidades que comparten los contenidos que son considerados de calidad.

\section{Los programas de entretenimiento des- tacan como el género con mayor presen- cia en la programación de los canales de televisión en España}

Ambas corrientes no se excluyen sino que son compatibles y se pueden combinar en una misma investigación. En Quality assessment of television, Ishikawa (1996) proponía un sistema objetivo de medición de la calidad en televisión que aúna la visión de los profesionales del medio y la de la audiencia. Sin embargo, para este autor el juicio de los profesionales tiene mayor peso que la opinión de la audiencia, que es sólo complementaria.

Estudios recientes también atienden al criterio profesional a la vez que incorporan la percepción de calidad de los te-

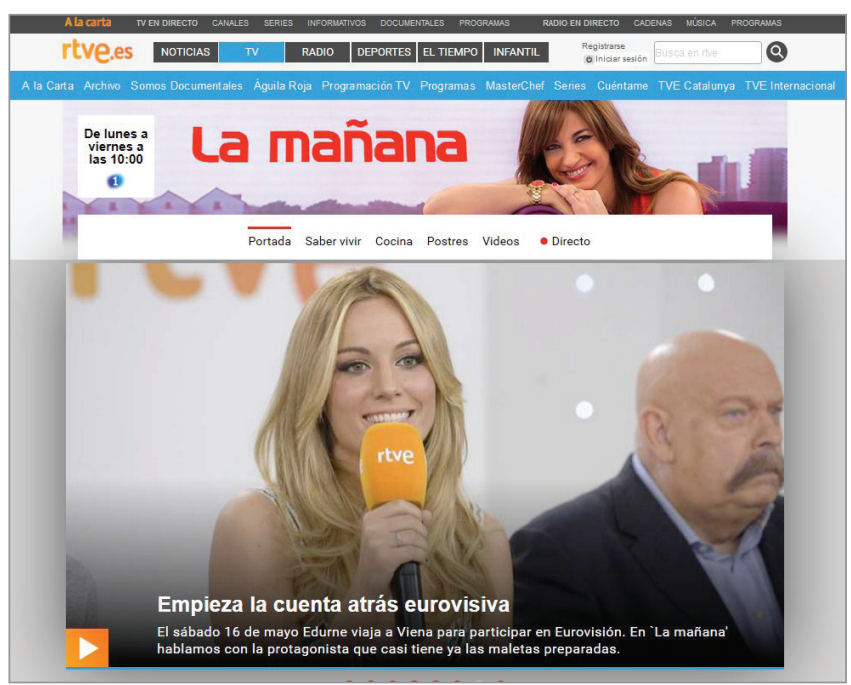

Magazine. La mañana, La 1

http://www.rtve.es/television/la-manana-de-la-1

lespectadores (Shamir, 2007; Mir et al., 2008; Artero; Herrero; Sánchez-Tabernero, 2010; Diego; Etayo; Pardo, 2011; Artero; Etayo; Sánchez-Tabernero; 2013). Más aún, comparan la percepción de la industria -en concreto operadores o proveedores de servicios y contenidos audiovisuales- con la de la audiencia (Pi-Tzong; Hsi-Peng; Tzu-Chuan, 2012).

Sin embargo es necesario subrayar el riesgo de confundir los resultados de audiencia derivados de la audimetría con la satisfacción o percepción de calidad. Un mayor consumo televisivo no implica ni mayor satisfacción ni mejor percepción de calidad por parte del público (Artero; Herrero; Sánchez-Tabernero, 2010). Más aún, los telespectadores admiten que en ocasiones les gusta ver determinados contenidos que califican como de baja calidad (Gunter; Wober, 1992; Shamir, 2007). Es imprescindible por tanto distinguir entre el nivel de satisfacción o la preferencia por determinados contenidos y la percepción de calidad.

Por último, son escasos los estudios que atienden a la percepción de calidad de la audiencia respecto a los valores de producción presentes en los contenidos. Si bien el público es capaz de mostrar su satisfacción con los diferentes programas -teoría de usos y gratificaciones (Palmgreen; Rayburn, 1985; Perse; Rubin, 1988)-, no es tan evidente su sensibilidad para reconocer los valores de producción (Shamir, 2007; Cummins; Chambers, 2011).

\section{Metodología}

Antes de definir la metodología se revisó la bibliografía académica más relevante en el ámbito de estudio-citada en el epígrafe anterior-. Las fuentes consultadas pueden clasificarse en tres áreas:

a) teoría de los usos y gratificaciones;

b) calidad en televisión y percepción de calidad;

c) valores de producción audiovisual y percepción de calidad.

Este trabajo se encuadra en esta última. En esta línea se vio la necesidad de realizar una encuesta original dirigida a la audiencia como fuente de información primaria, pues los datos que se requerían no podían ser facilitados directa- 
mente por la industria ni por los estudios precedentes. El método de encuesta ha sido ampliamente utilizado para el estudio de las audiencias (por ejemplo, Schweiger, 2000; Valkenburg et al., 2009).

Los resultados que se muestran en este artículo proceden de una encuesta propia realizada en 2012. El universo de referencia fue la población española de 14 años en adelante (40.606.471 habitantes). Se siguió un proceso polietápico para el diseño de la muestra, que se compuso de 1.000 personas, y fue estratificada por zona geográfica y por tamaño poblacional de las localidades. Para la selección de encuestados se optó por un criterio de cuotas de género y edad. La muestra estuvo compuesta por un 52,1\% de mujeres y un $47,9 \%$ de hombres y un $13,4 \%$ de personas entre 14 y 24 años, 36,5\% de entre 25 y 44, 30,1\% de entre 55 y 64 años y $20 \%$ de 65 o más años. Un 23,9\% vivían en el centro del país, $24,9 \%$ en el Sur, $19,3 \%$ en el Norte y $31,9 \%$ en el Este. Asimismo, un $31,6 \%$ vivían en capitales de provincia, $12,9 \%$ en municipios de menos de 5.000 habitantes, $34,9 \%$ en localidades de entre 5.000 y 50.000 y $20,6 \%$ en poblaciones de más de 50.000 habitantes.

El género preferido son las docuseries y el menos apreciado la telerrealidad

El margen de error fue del 3,1\% y el nivel de confianza del $95,5 \%$. Las entrevistas se realizaron mediante CATI (computed aided telephone interview), con una duración media de 15 minutos cada una. Se obtuvo un $18,4 \%$ de respuestas.

A continuación se analiza en qué medida los valores de producción influían en la percepción de calidad que la audiencia española tenía sobre los programas de entretenimiento emitidos por la televisión en 2012. Además hemos incluido otras variables necesarias para la correcta interpretación de los datos, como las preferencias por géneros, y algunos fac-

Tabla 2. Correlaciones de Spearman entre el gusto por los programas de entretenimiento ${ }^{2}$

\begin{tabular}{|l|l|c|c|c|c|c|}
\hline & Concursos & $\begin{array}{c}\text { Reality } \\
\text { shows }\end{array}$ & $\begin{array}{c}\text { Programas } \\
\text { musicales y } \\
\text { talent shows }\end{array}$ & $\begin{array}{c}\text { Programas de } \\
\text { humor y } \\
\text { entretenimiento }\end{array}$ & Divulgativos & Docuseries \\
\hline $\begin{array}{l}\text { Magazines y talk } \\
\text { shows }\end{array}$ & $0,178^{* * *}$ & $0,179^{* * *}$ & $0,151^{* * *}$ & $0,130^{* * *}$ & $0,173^{* * *}$ & $0,213^{* * *}$ \\
\hline Concursos & & $0,238^{* * *}$ & $0,368^{* * *}$ & $0,302^{* * *}$ & $0,279^{* * *}$ & $0,245^{* * *}$ \\
\hline Reality shows & & $0.256^{* * *}$ & $0,101^{* * *}$ & $0,063^{* *}$ & $0,108^{* * *}$ \\
\hline $\begin{array}{l}\text { Programas musica- } \\
\text { les y talent shows }\end{array}$ & & & $0,343^{* * *}$ & $0,284^{* * *}$ & $0,235^{* * *}$ \\
\hline $\begin{array}{l}\text { Programas de } \\
\text { humor y entreteni- } \\
\text { miento }\end{array}$ & & & & & $0,226^{* * *}$ & $0,210^{* * *}$ \\
\hline \begin{tabular}{l} 
Divulgativos \\
\hline
\end{tabular} & & & & & & $0,345^{* * *}$ \\
\hline
\end{tabular}

$* * * \mathrm{p}<0,01, * * \mathrm{p}<0,05,{ }^{*} \mathrm{p}<0,10$ tores pragmáticos y morales que pueden influir en la decisión de ver o no los contenidos.

\section{Resultados}

Los programas de entretenimiento destacan como el género con mayor presencia en la programación de los canales de televisión en España. Según Kantar Media, consultora que mide e investiga audiencias en el mercado televisivo español, en 2012 más del 35\% de los contenidos de entretenimiento programados pertenecían a esta categoría, mientras que en 2013 la cifra superó el 43\%. Además los datos de la audimetría televisiva confirman que también son los géneros más vistos, encabezados por los concursos. La tabla 1 muestra el gusto de los telespectadores por los programas de entretenimiento, agrupados en los géneros magazines y talk shows, concursos, telerrealidad (reality shows), programas musicales y de búsqueda de talentos (talent shows), programas de entretenimiento y humor, programas divulgativos y docuseries. En concreto, en relación con esta cuestión, al entrevistado se le preguntó: “¿cuánto le gustan los siguientes géneros televisivos?", debiendo indicar si nada, un poco, lo normal, bastante o mucho.

Los contenidos que más gustan a los entrevistados son docuseries, seguidos de programas de entretenimiento y humor. $A$ continuación van programas divulgativos, concursos, programas musicales y de búsqueda de talentos, y magazines y talk shows. El género que menos gusta es telerrealidad. A un 71,1\% de espectadores no les gusta nada los reality shows, mientras que el porcentaje a los que no les gusta nada el resto de contenidos desciende hasta alrededor del $30 \%$. Las docuseries gustan bastante o mucho al $66,3 \%$ de espectadores, los programas de entretenimiento y humor al $52,8 \%$, los divulgativos al $42,1 \%$, los concursos al $37,3 \%$, los musicales y de búsqueda de talentos al $35 \%$, y, finalmente, los magazines y talk shows al $32,8 \%$. 


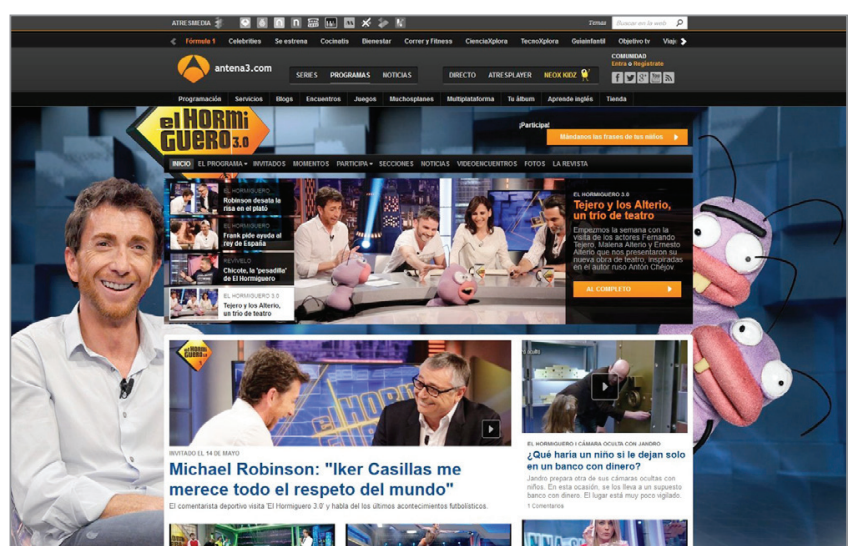

Entretenimiento y humor. El hormiguero, Antena 3

http://www.antena3.com/programas/el-hormiguero

Por tanto hay preferencias por géneros muy dispares. El género preferido, las docuseries, gusta bastante o mucho a casi dos tercios de los espectadores. Sin embargo la telerrealidad es el género menos apreciado, a considerable distancia del resto de contenidos de entretenimiento -sólo gusta bastante o mucho al $12,8 \%$.

La tabla 2 analiza la asociación entre la preferencia por un tipo de programa de entretenimiento y por el resto de contenidos de este género, a pesar de las acentuadas diferencias entre ellos en formato, guión, producción, etc. Para ello se emplea el coeficiente de correlación de Spearman, que sirve para analizar la asociación entre dos variables medidas en una escala ordinal, como es el caso (Newbold; Carlson; Thorne, 2007). Este coeficiente ha sido empleado con el mismo fin en otros trabajos de comunicación (Yagade; Dozier, 1990; Ku; Kaid; Pfau, 2003). El intervalo de valores que puede tomar es el comprendido entre -1 y 1 , ambos inclusive. El primer valor indica una asociación negativa perfecta, y el segundo una positiva perfecta. El valor cero indica ausencia de asociación.

La correlación entre preferencias por los tipos de programas analizados es muy alta. Hay una asociación significativa y positiva entre el gusto por el entretenimiento y el gusto por cada uno de los tipos de programas que satisfacen esta necesidad. La correlación es especialmente alta entre concursos y programas musicales y de búsqueda de talentos. También es importante la que existe entre estos dos géneros y los programas de entretenimiento y humor. Las corre-

Tabla 3. Preferencia por géneros de entretenimiento y percepción de calidad (correlaciones de Spearman) ${ }^{2}$

\begin{tabular}{|l|c|}
\hline \multicolumn{1}{|c|}{ Géneros } & Percepción de calidad \\
\hline Concursos & $0,321^{* * *}$ \\
\hline Programas musicales y talent shows & $0,251^{* * *}$ \\
\hline Programas de humor y entretenimiento & $0,233^{* * *}$ \\
\hline Divulgativos & $0,160^{* * *}$ \\
\hline Docuseries & $0,141^{* * *}$ \\
\hline Magazines y talk shows & $0,158^{* * *}$ \\
\hline Reality shows & $0,242^{* * *}$ \\
\hline
\end{tabular}

$* * * \mathrm{p}<0,01, * * \mathrm{p}<0,05, * \mathrm{p}<0,10$

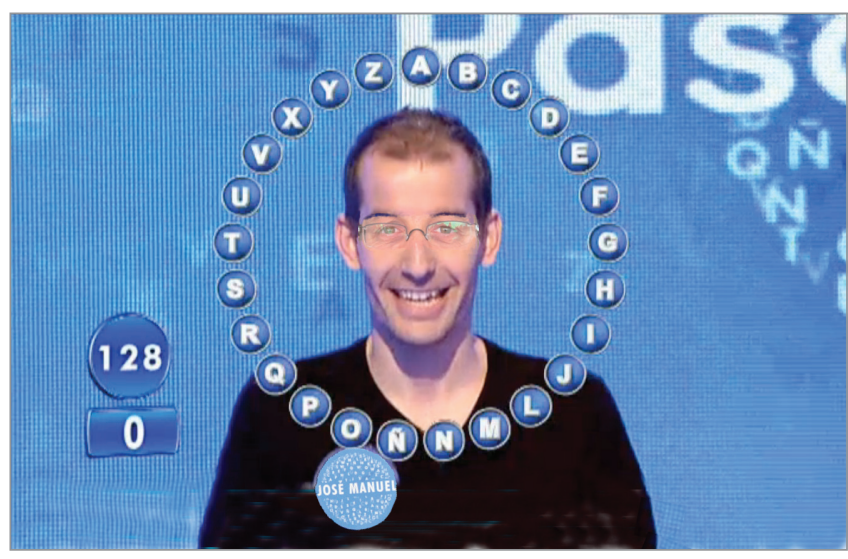

Concurso. Pasapalabra, Telecinco

http://www.telecinco.es/pasapalabra

laciones más débiles son entre reality shows y divulgativos, y es también baja entre telerrealidad y docuseries.

Es interesante analizar en qué medida la percepción de calidad de los programas de entretenimiento varía en función de la preferencia del público por los diferentes géneros. La pregunta concreta fue: “¿qué le parece la calidad de los programas de entretenimiento en televisión (concursos, realities, humor, etc.)?", debiendo elegir el entrevistado una entre las siguientes cinco opciones: muy mala, mala, regular, buena y muy buena.

Los datos de la tabla 3 muestran cómo el gusto por programas de entretenimiento está asociado a la creencia de que sus contenidos son de calidad. Aquellos a quienes les gustan mucho los concursos aprecian mayor calidad en los programas de entretenimiento. En un segundo grupo, en cuanto a su asociación con la creencia de que los contenidos de entretenimiento son de calidad, se encuentran quienes prefieren los programas musicales y de búsqueda de talentos, la telerrealidad y los programas de entretenimiento y humor. Por último la correlación es menor aunque estadísticamente significativa entre la calidad percibida y el gusto por los siguientes géneros: espacios divulgativos, magazines y talk shows y docuseries. Por tanto, los resultados apuntan a que los telespectadores a los que les gustan los programas de entretenimiento tienden a percibir en gran medida que sus contenidos son de calidad.

El principal objeto de estudio de este artículo es esclarecer en qué medida determinados valores de producción influyen o no en la percepción de calidad del telespectador. La tabla 4 contiene los datos referidos a esta cuestión. Se han seleccionado cuatro valores de producción que represen-

Tabla 4. Valores de producción y percepción de calidad de los programas de entretenimiento (correlaciones de Spearman) ${ }^{2}$

\begin{tabular}{|l|c|}
\hline \multicolumn{1}{|c|}{ Valores de producción } & Percepción de calidad \\
\hline Presentador/a & $0,514^{* * *}$ \\
\hline Contenido & $0,643^{* * *}$ \\
\hline Decorado & $0,324^{* * *}$ \\
\hline Calidad creativa y técnica & $0,425^{* * *}$ \\
\hline
\end{tabular}

$* * * p<0,01, * * p<0,05, * p<0,10$ 
Tabla 5. Preferencia por géneros de entretenimiento y preocupación por factores morales y pragmáticos (correlaciones de Spearman)²

\begin{tabular}{|c|c|c|c|c|c|c|c|}
\hline & $\begin{array}{l}\text { Magazines y } \\
\text { talk shows }\end{array}$ & Concursos & Reality shows & $\begin{array}{l}\text { Programas } \\
\text { musicales y } \\
\text { talent shows }\end{array}$ & $\begin{array}{l}\text { Programas de humor } \\
\text { y entretenimiento }\end{array}$ & Divulgativos & Docuseries \\
\hline $\begin{array}{l}\text { Incumplimiento del } \\
\text { horario de protección } \\
\text { infantil }\end{array}$ & $0,095^{* * *}$ & 0,008 & $-0,065^{* *}$ & 0,018 & $-0,008$ & $0,077^{* *}$ & $0,083^{* * *}$ \\
\hline Violencia & $0,079^{* *}$ & $0,088^{* * *}$ & $-0,059^{*}$ & 0,036 & $-0,087^{* * *}$ & $0,188^{* * *}$ & $0,141^{* * *}$ \\
\hline Erotismo y pornografía & 0,044 & $0,146^{* * *}$ & $-0,023$ & $0,121^{* * *}$ & $-0,070^{* *}$ & $0,138^{* * *}$ & $0,151^{* * *}$ \\
\hline Exceso de publicidad & 0,012 & $-0,025$ & $-0,087^{* * *}$ & $-0,079^{* *}$ & 0,016 & $-0,027$ & 0,017 \\
\hline $\begin{array}{l}\text { Falta de programación } \\
\text { cultural }\end{array}$ & $-0,011$ & 0,002 & $-0,185^{* * *}$ & $-0,075^{* *}$ & $-0,035$ & $0,105^{* * *}$ & $0,087^{* * *}$ \\
\hline Cotilleo & $-0,092^{* * *}$ & $-0,143^{* * *}$ & $-0,319^{* * *}$ & $-0,181^{* * *}$ & $-0,037$ & $-0,129 * * *$ & $-0,066^{* *}$ \\
\hline $\begin{array}{l}\text { Ausencia de variedad } \\
\text { en la programación }\end{array}$ & $-0,036$ & $-0,101^{* * *}$ & $-0,138^{* * *}$ & $-0,099^{* * *}$ & $-0,015$ & $-0,075^{* *}$ & 0,007 \\
\hline Sensacionalismo & $-0,032$ & $-0,109 * * *$ & $-0,196^{* * *}$ & $-0,180^{* * *}$ & $-0,056^{*}$ & $-0,016$ & $-0,049$ \\
\hline
\end{tabular}

$* * * \mathrm{p}<0,01, * * \mathrm{p}<0,05, * \mathrm{p}<0,10$

tan un elevado porcentaje del presupuesto de producción y que son fácilmente reconocibles por la audiencia durante el visionado: contenidos, presentadores, decorados y calidad técnica y creativa. La pregunta exacta fue: “¿qué le parecen en general los presentadores/el contenido/los decorados/ la calidad técnica y creativa de los programas de entretenimiento en televisión?". Las posibles respuestas son: muy malos, malos, regular, buenos y muy buenos.

El factor más asociado a la percepción de calidad de un programa es el contenido: si el contenido es bueno, se percibe que el programa es de buena calidad, al igual que ocurre en el mercado de bienes tangibles. Este dato confirma que el contenido es la clave de toda industria creativa, independientemente de los cambios que impliquen las innovaciones tecnológicas.

El contenido es la clave de toda industria creativa, independientemente de los cambios que impliquen las innovaciones tecnológicas

En segundo lugar se encuentran los presentadores. Elegir al presentador con el perfil adecuado para el programa es otro factor de relevancia para que éste sea percibido como un producto de calidad. Si bien este dato aislado no confirma la existencia de un star system televisivo de contenidos de entretenimiento, sí que sobresale como un valor de producción muy preciado, recordándonos que el talento destaca como un factor crucial en el sector audiovisual.

En tercer lugar la audiencia valora la innovación en todos los aspectos relacionados con la calidad técnica -el modo en que está audiovisualmente realizado el programa- y la calidad creativa -formato, guión, tratamiento de los contenidos, puesta en escena, etc.-.

Por último, y aunque todos los factores considerados influyen en la percepción de calidad de los programas de entretenimiento, los decorados son el factor que menos repercusión tiene. Se evidencia que un buen decorado se asocia a una evaluación positiva del programa, aunque entre los cuatro factores analizados, es el que tiene una menor vinculación con la percepción de calidad.

Un trabajo de investigación sobre percepción de calidad de contenidos audiovisuales no estaría completo si no se recogieran las principales preocupaciones que muestra la audiencia con respecto a esos contenidos. En la tabla 5 quedan recogidas las correlaciones existentes entre la preferencia de los telespectadores por los distintos programas de entretenimiento y la preocupación que declaran por algunos aspectos de tipo moral (incumplimiento del horario infantil, contenidos violentos, y erotismo y pornografía). Se analizan también las correlaciones entre el gusto mostrado por los distintos géneros de entretenimiento y factores de orden pragmático (exceso de publicidad, ausencia de programas culturales, exceso de programas de cotilleo, falta de variedad en la programación y sensacionalismo). La manera de medir la preocupación por estos factores, tanto morales como pragmáticos, fue a través de la pregunta: "Indique cuánto le molestan los siguientes aspectos relacionados con los contenidos televisivos". Las posibles respuestas eran: nada, poco, regular, bastante y mucho.

Los resultados necesitan ser interpretados género a género para obtener conclusiones firmes; no obstante, pueden extraerse algunas consideraciones generales.

El gusto por los magazines y talk shows, los programas divulgativos y las docuseries está unido a una mayor molestia por el incumplimiento del horario de protección infantil. En contraposición, los telespectadores que prefieren los títulos de telerrealidad no se sienten preocupados en absoluto por esta cuestión. Los contenidos violentos molestan a aquellos a los que les gustan los géneros anteriormente mencionados y también a aquellos a los que les gustan los concursos. Sin embargo, de nuevo la preferencia por los reality shows y por los programas de entretenimiento y humor, no está asociada a una sensación de molestia por la presencia de contenidos violentos.

Por su parte, el erotismo y la pornografía son más molestos conforme mayor es la preferencia por los concursos, los programas musicales y de búsqueda de talentos, los divulgati- 


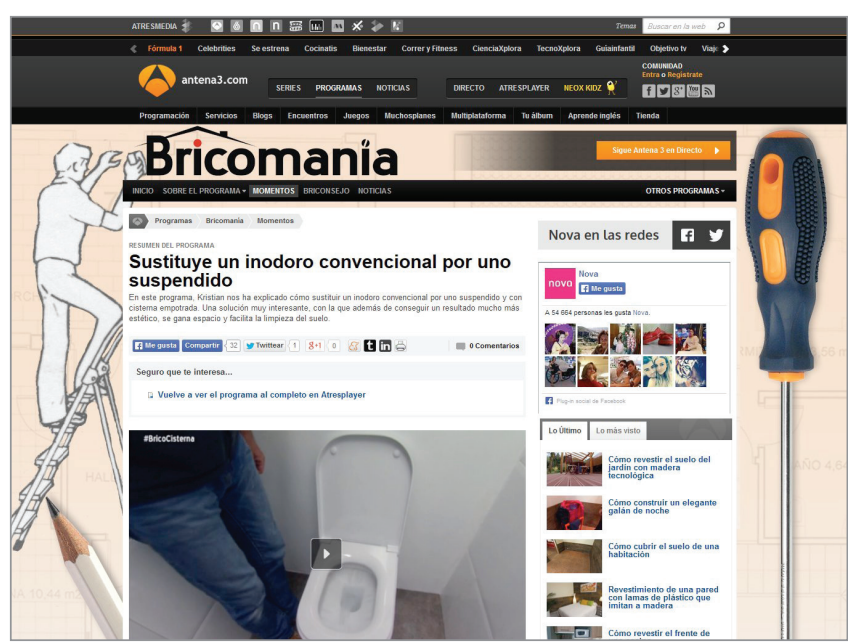

Divulgativos. Bricomania, Nova

http://www.antena3.com/programas/bricomania

vos y las docuseries. La relación contraria se ha hallado para los espacios de entretenimiento y humor.

Respecto al exceso de publicidad, a grandes rasgos no es una molestia asociada al gusto por los títulos de entretenimiento. De hecho, el trabajo de Etayo (2014) muestra la existencia de correlación entre la preferencia por programas de entretenimiento y el gusto por la publicidad. Más aún, a quienes les gustan la telerrealidad y los programas musicales y talent shows no les molesta en absoluto el exceso de publicidad. A estos últimos además no les preocupa nada la ausencia de programas culturales, que sí es motivo de preocupación para los que muestran preferencia por los espacios divulgativos y las docuseries.

Los telespectadores a los que les gustan los géneros de entretenimiento no se sienten molestos por la emisión de programas sobre la vida íntima de personajes populares (cotilleo), con la excepción de quienes prefieren los programas de entretenimiento y humor -no se encuentra relación-. La falta de variedad en la programación no es motivo de preocupación para los aficionados a programas de entretenimiento. La correlación es negativa y significativa con excepción de los que prefieren magazines y talk

Tabla 6. Correlación entre la calidad percibida de los programas de entretenimiento y la preocupación por factores morales y pragmáticos (correlaciones de Spearman) ${ }^{2}$

\begin{tabular}{|l|c|}
\hline \multicolumn{1}{|c|}{ Factores morales y pragmáticos } & Quality perception \\
\hline $\begin{array}{l}\text { Incumplimiento del horario de protección } \\
\text { infantil }\end{array}$ & $-0,082^{* *}$ \\
\hline Violencia & $-0,027$ \\
\hline Erotismo y pornografía & $-0,006$ \\
\hline Exceso de publicidad & $-0,050$ \\
\hline Falta de programación cultural & $-0,134^{* * *}$ \\
\hline Cotilleo & $-0,178^{* * *}$ \\
\hline Ausencia de variedad en la programación & $-0,174^{* * *}$ \\
\hline Sensacionalismo & $-0,216^{* * *}$ \\
\hline
\end{tabular}

${ }^{* * *} p<0,01, * * p<0,05, * p<0,10$ shows, programas de entretenimiento y humor y docuseries. Tampoco es causa de molestia el sensacionalismo, que está correlacionado negativamente con la preferencia por géneros como los concursos, la telerrealidad, los programas musicales y de búsqueda de talentos, y los de humor y entretenimiento.

Finalmente, en la tabla 6 se muestra la relación existente entre la calidad percibida de los programas de entretenimiento en general -sin distinción de géneros- y la preocupación que la audiencia declara respecto a los problemas morales y pragmáticos antes enumerados. A quien le molesta el incumplimiento del horario infantil, la falta de programación cultural, el cotilleo, la ausencia de variedad en la programación y el sensacionalismo en el tratamiento de los contenidos, le parece que la calidad de los géneros de entretenimiento es menor. Sin embargo, la molestia originada por los contenidos violentos, el erotismo y la pornografía y el exceso de publicidad no está vinculada a la percepción de calidad de los programas de entretenimiento (la correlación no es significativa).

Sensacionalismo, contenidos de cotilleo, ausencia de variedad en la programación, falta de programación cultural e incumplimiento del horario de protección infantil son los factores de mayor impacto negativo

\section{Conclusiones}

Valorar la percepción de calidad por parte de la audiencia de un contenido audiovisual es una tarea compleja, pues depende de factores muy diversos y no todos son controlables por el sector profesional, como ocurre con los culturales. No obstante, una de las variables que influyen en la percepción de calidad sí que depende directamente de la industria: los valores de producción presentes en el producto. Los resultados confirman la hipótesis de partida, y demuestran que todos los valores de producción influyen en la percepción de calidad de la audiencia, aunque destaca sobre todo el contenido. Esto indica que la percepción de calidad está estrechamente vinculada con la calidad del contenido más que con otros valores de producción como el presentador, el decorado o la calidad técnica, si bien todos son relevantes.

A pesar de que el público carezca de criterio profesional, asocia un mayor estándar de producción a contenidos de mejor calidad. Más aún, los telespectadores de casi todos los géneros de entretenimiento reconocen que se sienten molestos con ciertos aspectos de orden moral como la falta de respeto al horario de protección infantil, la emisión de contenidos violentos, y el erotismo o la pornografía -excepto los telespectadores de reality shows y de programas de humor y entretenimiento ligero.

El sensacionalismo, el exceso de contenidos de cotilleo sobre la vida privada de personajes, la ausencia de variedad en la programación, la falta de programación cultural y el incumplimiento del horario de protección infantil destacan 


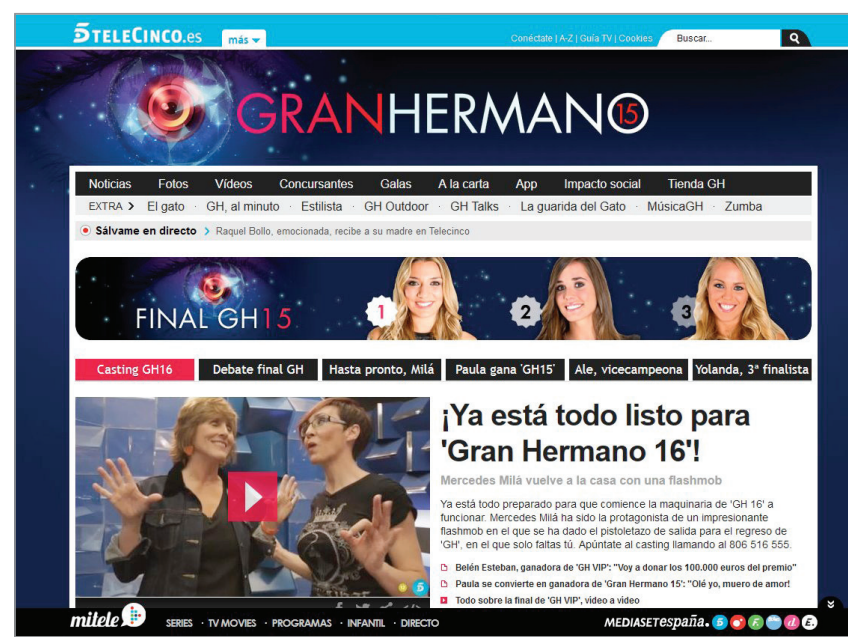

Telerrealidad. Gran hermano, Telecinco http://www.telecinco.es/granhermano

como los factores que tienen un mayor impacto negativo sobre la percepción de calidad.

Es importante remarcar que para valorar adecuadamente esta percepción de calidad es necesario identificar primero cuáles son los valores estándar o los patrones de referencia de la población respecto a un producto de calidad. Diferentes escalas de valores dan como resultado juicios dispares sobre calidad (Shamir, 2007). Esta circunstancia se cumple no sólo en el caso de la audiencia, sino también en el de los profesionales, descubriendo un área en la que es necesario profundizar en futuras investigaciones.

La esencia del negocio, la creatividad, no se ha visto alterada

Para finalizar, aunque la encuesta es el método más adecuado para recoger las opiniones y actitudes de los telespectadores, no por ello está exenta de limitaciones. Esto es especialmente cierto cuando se abordan cuestiones sensibles en las que puede aparecer un sesgo de deseabilidad social. En este caso existe el riesgo de que al ser preguntados por las preferencias por determinados géneros polémicos como la telerrealidad, los entrevistados hayan tendido a contestar de manera que sus respuestas se ajusten no tanto a su verdadera opinión, sino a lo que ellos entienden que es lo valorado de forma positiva socialmente. De aquí que no exista una relación directa entre los resultados de este estudio y los índices de audiencia.

En definitiva, pese a los cambios sustanciales motivados por la innovación tecnológica que ha experimentado la industria audiovisual, y en concreto la televisiva, la esencia del negocio no se ha visto alterada: la creatividad. La apuesta por un contenido de calidad como valor de producción mejora la percepción de calidad y, por tanto, contribuye a cuidar la imagen corporativa de emisores y productores, al tiempo que sirve de garantía frente a los riesgos asociados al sector audiovisual. Ofrecer contenidos de calidad siempre es una decisión acertada y bien valorada por la audiencia.

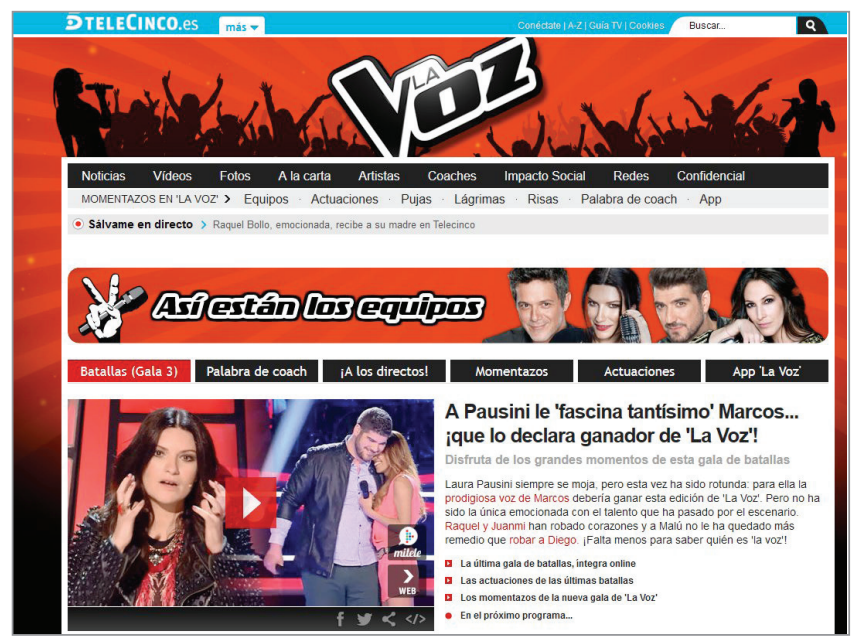

Búsqueda de talentos. La voz, Telecinco http://www.telecinco.es/lavoz

\section{Notas}

1. A modo de ejemplo se incluye un título de entretenimiento de cada género: concursos (Pasapalabra, Telecinco), magazines y talk shows (La mañana, La 1), telerrealidad (Gran hermano, Telecinco), programas musicales y de búsqueda de talentos (La voz, Telecinco), programas de entretenimiento y humor (El hormiguero, Antena 3), divulgativos (Bricomanía, Nova) y docuseries (Un país para comérselo, La 1).

2. Sólo llevan asterisco las cantidades que corresponden a relaciones entre variables estadisticamente significativas. Las que no llevan es porque la relación entre variables no lo es.

Un asteristico corresponde a significatividad al 90\%, dos al $95 \%$ y tres supone que es significativa al $99 \%$.

3. Este artículo forma parte de dos proyectos de investigación subvencionados: Los nuevos hábitos de consumo en los contenidos audiovisuales: el impacto de la digitalización en la dieta de medios europeos, financiado por el Ministerio de Economía y Competitividad del período 2011-14 (CSO201020122); y El impacto de la digitalización en la industria audiovisual española (2011-13), financiado por la Universidad de Navarra (Piuna).

Todos los valores de producción influyen en la percepción de calidad de la audiencia, aunque destaca sobre todo el contenido

\section{Bibliografía}

Albers, Robert (1992). "Quality in television from the perspective of the professional program maker". Studies of broadcasting, n. 28, pp. 7-75.

Artero, Juan-Pablo; Etayo, Cristina; Sánchez-Tabernero, Alfonso (2013). "Effects of pragmatic and moral concerns on perceived TV quality". Journal of applied journalism \& media studies, v. 2, n. 3, pp. 489-504.

http://dx.doi.org/10.1386/ajms.2.3.489_1 
Artero, Juan-Pablo; Herrero, Mónica; Sánchez-Tabernero, Alfonso (2010). "La calidad de la oferta televisiva en el mercado español: las percepciones del público". Zer, v. 15, n. 28, pp. 49-63.

http://www.ehu.eus/zer/hemeroteca/pdfs/zer28-03artero.pdf

Cummis, R. Glenn; Chambers, Todd (2011). "How production value impacts perceived technical quality, credibility, an economic value of video news". Journalism \& mass communication quaterly, v. 88, n. 4, pp. 737-752.

https://goo.gl/ZxXjVy

Diego, Patricia; Etayo, Cristina; Pardo, Alejandro (2011). "La percepción sobre la calidad de las series televisivas en España: contraste entre el público y los profesionales". Zer, v. 16, n. 31, pp. 69-88.

http://www.ehu.eus/zer/hemeroteca/pdfs/zer31-04-diego.pdf

Diego, Patricia; Pardo, Alejandro (2008). “Estándares de producción de 'dramedias' familiares en España: el caso de Médico de familia, Cuéntame cómo pasó y Los Serrano". En: Medina, Mercedes (2008). Series de televisión: el caso de Médico de familia, Cuéntame cómo pasó y Los Serrano. Madrid: Eiunsa, pp. 45-74. ISBN: 9788484692423

Etayo, Cristina (2014). "Géneros preferidos y actitud hacia la publicidad". Questiones publicitarias, v. 1, n. 19, pp. 64-81. http://www.maecei.es/pdf/n19/articulos/A5-Generostelevisivos-preferidos-y-actitudes-hacia-la-publicidad.pdf

Guerrero, Enrique (2005). "El concurso como género de calidad en la televisión española". Comunicar, v. 13, n. 25. http://dialnet.unirioja.es/servlet/articulo?codigo $=2927584$

Guerrero, Enrique (2013). Guión y producción de programas de entretenimiento. Pamplona: Eunsa. ISBN: 978 8431328962

Gunter, Barrie; Wober, Mallory (1992). The reactive viewer: Review of research on audience reaction measurement (Independent Television Commission Research Monograph). Londres: John Libbey. ISBN: 9780861963584

Gutiérrez-Gea, Charo (2000). "Televisión y calidad: perspectivas de investigación y criterios de evaluación”. Zer, v. 5, n. 9, pp. 151-184.

http://www.ehu.eus/zer/hemeroteca/pdfs/zer09-06gutierrez.pdf

Ishikawa, Sakae (1996). Quality assessment of television. Luton: University of Luton Press. ISBN: 1860205070

Kantar Media (2012-2013). Datos de audimetría. http://www.kantarworldpanel.com

Ku, Gyotae; Kaid, Linda L.; Pfau, Michael (2003). "The impact of web site campaigning on traditional news media and public information processing". Journalism \& mass communication quarterly, v. 80, n. 3, pp. 528-547.

http://dx.doi.org/10.1177/107769900308000304

Lasagni, María-Cristina; Richeri, Giuseppe (2006). Televisión y calidad: el debate internacional. Buenos Aires: La Crujía. ISBN: 9789876010030
Medina, Mercedes (2009). Calidad y contenidos audiovisuales. Pamplona: Eunsa. ISBN: 9788431324247

Mir, M. Angélica; Errázuriz, M. Ignacia; Kimber, David; Santa-María, Isabel (2008). "Una propuesta para medir la calidad en la televisión en abierto". Revista de comunicación, n. 7, pp. 56-80.

Newbold, Paul; Carlson, William L.; Thorne, Betty M. (2007). Estadística para administración y economía. Madrid: Pearson Prentice Hall. ISBN: 9788483224038

Palmgreen, Philip; Rayburn, James D. (1985). “A comparison of gratification models of media satisfaction". Communication monographs, v. 52, n. 4, pp. 334-46.

http://files.eric.ed.gov/fulltext/ED247575.pdf

Perse, Elizabeth M.; Rubin, Alan M. (1988). "Audience activity and satisfaction with favorite television soap opera". Journalism quarterly, v. 65, n. 2, pp. 368-75.

http://goo.gl/3GCkdO

Pi-Tzong, Jan; Hsi-Peng, Lu; Tzu-Chuan, Chou (2012). “Measuring the perception discrepancy of the service quality between provider and customers in the internet protocol television industry". Total quality management, v. 23, n. 8, pp. 981-995.

http://dx.doi.org/10.1080/14783363.2012.661135

Sánchez-Tabernero, Alfonso (2008). Los contenidos de los medios de comunicación. Calidad, rentabilidad y competencia. Barcelona: Deusto. ISBN: 9788423426133

Schweiger, Wolfwang (2000). “Media credibility - Experience or image. A survey on the credibility of the World Wide Web in Germany in comparison to other media". European journal of communication, v. 15, n. 1, pp. 37-59.

http://citeseerx.ist.psu.edu/viewdoc/download?doi=10.1.1. 201.3473\&rep $=$ rep1\&type $=p d f$

http://dx.doi.org/10.1177/0267323100015001002

Shamir, Jacob (2007). "Quality assessment of television programs in Israel: Can viewers recognize production value?". Journal of applied communication research, v. 35, n. 3, pp. 320-341.

http://dx.doi.org/10.1080/00909880701434406

Valkenburg, Patti M.; Krcmar, Marina; Peeters, Allerd L.; Marseille, Nies M. (1999). "Developing a scale to assess three styles of television mediation: 'instructive mediation', 'restrictive mediation' and 'social coviewing'". Journal of broadcasting \& electronic media, v. 43, n. 1, pp. 52-66. http://dx.doi.org/10.1080/08838159909364474

Von-Rimscha, M. Björn; De-Acevedo, Miriam; Siegert, Gabriele (2010). "Securing quality in public service television entertainment". Studies in communication sciences, v. 10, n. 2, pp. 7-25.

http://www.zora.uzh.ch/38884

Yagade, Aileen; Dozier, David M. (1990). "The media agenda-setting effect of concrete versus abstract issues". Journalism \& mass communication quarterly, v. 67, n. 1, pp. 3-10. http://dx.doi.org/10.1177/107769909006700102 